\title{
Two-step nilpotent Leibniz algebras ${ }^{12}$
}

\author{
Gianmarco La Rosa ${ }^{3}$ \\ Dipartimento di Matematica e Informatica \\ Università degli Studi di Palermo, Via Archirafi 34, 90123 Palermo, Italy \\ gianmarco.larosa@unipa.it \\ Manuel Mancini ${ }^{4}$ \\ Dipartimento di Matematica e Informatica \\ Università degli Studi di Palermo, Via Archirafi 34, 90123 Palermo, Italy \\ manuel.mancini@unipa.it
}

\begin{abstract}
In this paper we give a complete classification of two-step nilpotent Leibniz algebras in terms of Kronecker modules associated with pairs of bilinear forms. In particular, we describe the complex and the real case of the indecomposable Heisenberg Leibniz algebras as a generalization of the classical $(2 n+1)$-dimensional Heisenberg Lie algebra $\mathfrak{h}_{2 n+1}$. Then we use the Leibniz algebras - Lie local racks correspondence proposed by S. Covez to globally integrate the two-step nilpotent Leibniz algebras. As an application, we integrate the indecomposable nilpotent real Leibniz algebras with one-dimensional commutator ideal. We also show that every Lie quandle integrating a Leibniz algebra is induced by the conjugation of a Lie group and the Leibniz algebra is the Lie algebra of that Lie group.
\end{abstract}

\section{Introduction}

Leibniz algebras were first introduced by J.-L. Loday in [1] as a non-antisymmetric version of Lie algebras, and many results of Lie algebras were also established in Leibniz algebras. Earlier, such algebraic structures had been considered by A. Blokh, who called them D-algebras [2]. Leibniz algebras play a significant role in different areas of mathematics and physics.

In [3] and [4] Lie algebras with small dimensional commutator ideals have been considered. In this paper our main goal is to classify all the two-step nilpotent Leibniz algebras over a field of characteristic different from 2 .

The first Section is devoted to some background material on Leibniz algebras which will be useful for the rest of the paper. We refer the reader to [5] for more details.

In the second Section we use the definition of Kronecker module to classify all the indecomposable nilpotent Leibniz algebras with one-dimensional commutator ideal. We associate with each of them a pair of bilinear forms, one symmetric and one skew-symmetric, and we use the simultaneous reduction of this pair to show that there are only three classes of nilpotent Leibniz algebras with one-dimensional commutator ideal. Up to isomorphisms, the first class is determined by the companion matrix of the power of a monic irreducible polynomial, and the other two are unique. In this way, we give the definition of Heisenberg Leibniz algebra, Kronecker Leibniz algebra and Dieudonné Leibniz algebra.

In Section 3 we study the complex and the real case of the indecomposable Heisenberg Leibniz algebras. In this case, when the filed is $\mathbb{C}$, it is more convenient to use the Jordan canonical form of the companion matrix of the polynomial $(x-a)^{k}$, that is a $k \times k$ Jordan block of eigenvalue $a$. Moreover, when the dimension is 3 , we determine all the isomorphism classes of these algebras.

In the last Section we give a global answer to the coquecigrue problem for the two-step nilpotent Leibniz algebras. We mean the problem, formulated by J.-L. Loday [1], of finding a generalization of the Lie third theorem, which associates a local Lie group with any (real or complex) Lie algebra, to Leibniz algebras. That is, given any Leibniz algebra $\mathfrak{g}$, one wants to find a manifold endowed of an algebraic map which plays the role of Ad for Lie groups, and such that the tangent space at a distinguished point, endowed of the differential ad

\footnotetext{
${ }^{1}$ Keywords: Leibniz algebras, Nilpotent Leibniz algebras, Lie racks, Coquegigrue problem

${ }^{2}$ AMS MSC 17A32, 22A30, 20M99

${ }^{3}$ Supported by University of Palermo

${ }^{4}$ Supported by University of Palermo
} 
of Ad, is a Leibniz algebra isomorphic to $\mathfrak{g}$.

We prove that, if a Lie rack $R$ integrating a left Leibniz algebra $\mathfrak{g}$ is idempotent, then $\mathfrak{g}$ is a Lie algebra and the multiplication of $R$ is induced by the conjugation of a Lie group integrating $\mathfrak{g}$. Moreover, we want to show that the integration of two-step nilpotent Leibniz algebras is global. As an application, we describe the Lie global racks integrating the indecomposable nilpotent Leibniz algebras with one-dimensional commutator ideal classified in the second section.

\section{Preliminaries}

We assume that $\mathbb{F}$ is a field with $\operatorname{char}(\mathbb{F}) \neq 2$. For the general theory we refer to [5].

Definition 1.1. A left Leibniz algebra over $\mathbb{F}$ is a vector space $L$ over $\mathbb{F}$ endowed of a bilinear map (called commutator or bracket) $[-,-]: L \times L \rightarrow L$ which satisfies the left Leibniz identity

$$
[x,[y, z]]=[[x, y], z]+[y,[x, z]] \forall x, y, z \in L .
$$

In the same way we can define a right Leibniz algebra, using the right Leibniz identity

$$
[[x, y], z]=[[x, z], y]+[x,[y, z]] \forall x, y, z \in L .
$$

A Leibniz algebra that is both left and right is called symmetric Leibniz algebra. From now on we assume that $\operatorname{dim}_{\mathbb{F}} L<\infty$.

An equivalent way to define a left Leibniz algebra, is to say that the (left) adjoint $\operatorname{map} \operatorname{ad}_{x}=[x,-]$ is a derivation, for every $x \in L$. Clearly every Lie algebra is a Leibniz algebra and every Leibniz algebras with skewsymmetric commutator is a Lie algebra. Thus it is defined an adjunction (see [6]) between the category LieAlg of the Lie algebras and the category LeibAlg of the Leibniz algebras. The left adjoint of the functor inclusion $i:$ LieAlg $\rightarrow$ LeibAlg is the functor $\pi:$ LeibAlg $\rightarrow$ LieAlg that associates with every Leibniz algebra $L$ the quotient $L / \operatorname{Leib}(L)$, where $\operatorname{Leib}(L)=\operatorname{Span}_{\mathbb{F}}\{[x, x] \mid x \in L\}$. We observe that $\operatorname{Leib}(L)$ is the smallest bilateral ideal of $L$ such that $L / \operatorname{Leib}(L)$ is a Lie algebra. Moreover $\operatorname{Leib}(L)$ is an abelian Lie algebra.

As in the case of Lie algebras, a derivation of a Leibniz algebra is a linear map $d: L \rightarrow L$ such that

$$
d([x, y])=[d(x), y]+[x, d(y)] \forall x, y \in L .
$$

The left multiplications are particular derivations called inner derivations. With the usual bracket $\left[d_{1}, d_{2}\right]=d_{1} \circ d_{2}-d_{2} \circ d_{1}$, the set $\operatorname{Der}(L)$ is a Lie algebra and the set $\operatorname{Inn}(L)$ of all inner derivations of $L$ is an ideal of $\operatorname{Der}(L)$. Furthermore, $\operatorname{Aut}(L)$ is a Lie group and the associated Lie algebra is $\operatorname{Der}(L)$.

We define the left and the right center of a Leibniz algebra

$$
\mathrm{Z}_{l}(L)=\{x \in L \mid[x, L]=0\}, \mathrm{Z}_{r}(L)=\{x \in L \mid[L, x]=0\},
$$

and we observe that they coincide when $L$ is a Lie algebra. The center of $L$ is $\mathrm{Z}(L)=\mathrm{Z}_{l}(L) \cap \mathrm{Z}_{r}(L)$. In the case of symmetric Leibniz algebras, the left center and the right center are bilater ideals, but in general $\mathrm{Z}_{l}(L)$ is an ideal of the left Leibniz algebra $L$, meanwhile the right center is not even a subalgebra.

Definition 1.2. Let $L$ be a left Leibniz algebra over $\mathbb{F}$ and let

$$
L^{(0)}=L, L^{(k+1)}=\left[L, L^{(k)}\right], \forall k \geq 0,
$$

be the lower central series of $L$. $L$ is $n$-step nilpotent if $L^{(n-1)} \neq 0$ and $L^{(n)}=0$.

One can directly prove the following.

Proposition 1.3. If $L$ is a left two-step nilpotent Leibniz algebra, then $L^{(1)}=[L, L] \subseteq \mathrm{Z}(L)$ and $L$ is symmetric.

Proposition 1.4. If $L$ is a left nilpotent Leibniz algebra with $\operatorname{dim}_{\mathbb{F}} L^{(1)}=1$, then $L^{(1)} \subseteq \mathrm{Z}(L)$ and $L$ is symmetric. 


\section{Nilpotent Leibniz algebras with one-dimensional commutator ideal}

Let $L$ be a two-step nilpotent Leibniz algebra, and let $\left\{z_{1}, \ldots, z_{t}\right\}$ be a basis of the commutator ideal $[L, L]$, thus, for any $x, y \in L$,

$$
[x, y]=\phi_{1}(x, y) z_{1}+\cdots+\phi_{t}(x, y) z_{t}
$$

where $\phi_{i}: L \times L \rightarrow \mathbb{F}$ is a bilinear form, for every $i=1, \ldots, t$. In the Lie algebra case, $\phi_{1}, \ldots, \phi_{t}$ are skewsymmetric forms. In this section we reduce such a given bilinear form to a suitable canonical representation.

Let $L$ be a nilpotent Leibniz algebra with one-dimensional commutator ideal $[L, L]=\operatorname{Span}_{\mathbb{F}}\{z\}$, where $z \in L$ is fixed, and let $[x, y]=\phi(x, y) z$, where $\phi: L \times L \rightarrow \mathbb{F}$ is a bilinear form. We observe that, if $L$ is not a Lie algebra, then $\operatorname{Leib}(L)=[L, L] \subseteq \mathrm{Z}(L)$.

We can decompose $\phi$ into its symmetric and skew-symmetric parts

$$
\sigma=\frac{\phi+\phi^{t}}{2}, \quad \alpha=\frac{\phi-\phi^{t}}{2} .
$$

A complete classification of this pair of bilinear forms gives a classification of this class of Leibniz algebras. The simultaneous reduction to canonical form of a pair of bilinear forms was initiated by Kronecker in [7] and then was studied by J. Dieudonné in [8] .

Definition 2.1. A Kronecker module is a quadruple $\left(V_{1}, V_{2}, f_{1}, f_{2}\right)$, where $V_{1}, V_{2}$ are vector spaces over $\mathbb{F}$ and $f_{1}, f_{2}: V_{1} \rightarrow V_{2}$ are linear maps.

Every Kronecker module is the direct sum of indecomposable modules and these were completely classified by several authors (cf. [9]). Given a pair of bilinear forms $\alpha, \sigma$ defined over a Leibniz algebra $L$ as above, it is possible to associate the Kronecker module $\left(L, L^{*}, \bar{\alpha}, \bar{\sigma}\right)$, where $L^{*}$ is the algebraic dual space of $L$ and, for every $x, y$ in $L, \bar{\alpha}(x)$ and $\bar{\sigma}(y)$ are defined by

$$
\bar{\alpha}(x): z \mapsto \alpha(x, z), \quad \bar{\sigma}(y): z \mapsto \sigma(y, z), \forall z \in L .
$$

We say that $L$ is decomposable if $L=U \oplus U^{\perp}$, where $U^{\perp}$ is the orthogonal space of $U$ with respect to both $\alpha$ and $\sigma$. In this case it is possible to find a basis of $L$ such that the matrix associated with $\phi$ is a diagonal block matrix. With standard arguments we can assume that $L$ is indecomposable.

The indecomposable modules $\left(L, L^{*}, \bar{\alpha}, \bar{\sigma}\right)$ turn out to be one of the following three pairs

$$
\begin{aligned}
& \left(\begin{array}{cc}
0 & I_{n} \\
-I_{n} & 0
\end{array}\right),\left(\begin{array}{cc}
0 & A \\
A^{t} & 0
\end{array}\right) \\
& \left(\begin{array}{cc}
0 & A \\
-A^{t} & 0
\end{array}\right),\left(\begin{array}{cc}
0 & I_{n} \\
I_{n} & 0
\end{array}\right) \\
& \left(\begin{array}{cc}
0 & J_{1} \\
-J_{1} & 0
\end{array}\right),\left(\begin{array}{cc}
0 & J_{2} \\
J_{2}^{t} & 0
\end{array}\right)
\end{aligned}
$$

where $A \in \mathrm{M}_{n}(\mathbb{F})$, and $J_{1}, J_{2}$ are the matrices associated with linear applications $F_{1}, F_{2}: \mathbb{F}^{n} \rightarrow \mathbb{F}^{n+1}$ defined by $F_{1}\left(x_{1}, \ldots, x_{n}\right)=\left(x_{1}, \ldots, x_{n}, 0\right)$ and $F_{2}\left(x_{1}, \ldots, x_{n}\right)=\left(0, x_{1}, \ldots, x_{n}\right)$.

The following result is a useful tool for the classification of the indecomposable nilpotent Leibniz algebras with one-dimensional commutator ideals. .

Proposition 2.2. Let $L_{1}$ and $L_{2}$ be Leibniz algebras of dimension $n$ with one-dimensional commutator ideals $\left[L_{1}, L_{1}\right]=\mathbb{F} z_{1}$ and $\left[L_{2}, L_{2}\right]=\mathbb{F} z_{2}$, and let $\phi_{1}$ and $\phi_{2}$ be the bilinear forms associated with $L_{1}$ and $L_{2}$ respectively. Let $\Phi_{1}$ and $\Phi_{2}$ be the matrices of $\phi_{1}$ and $\phi_{2}$ respectively. $L_{1}$ is isomorphic to $L_{2}$ if and only if $\Phi_{1}$ is congruent to $\Phi_{2}$. 
Proof. Let $\varphi: L_{1} \rightarrow L_{2}$ be a Leibniz algebras isomorphism and let $\left\{e_{1}, \ldots, e_{n-1}, z_{1}\right\}$ be a basis of $L_{1}$. Then $\varphi\left(z_{1}\right)=k z_{2}$, for some $k \in \mathbb{F}^{*}$, and $\left\{\varphi\left(e_{1}\right), \ldots, \varphi\left(e_{n-1}\right), k z_{2}\right\}$ is a basis of $L_{2}$ such that the associated matrix is $\Phi_{1}$. Then there exists a matrix $P \in \mathrm{GL}_{n}(\mathbb{F})$ such that $P \Phi_{2} P^{t}=\Phi_{1}$.

Conversely, we suppose that there exists $P \in \mathrm{GL}_{n}(\mathbb{F})$ such that $P \Phi_{1} P^{t}=\Phi_{2}$. $\Phi_{1}$ and $\Phi_{2}$ are matrices associated with bilinear forms, so $P$ induces a change of basis $\left\{e_{1}, \ldots, e_{n-1}, z_{1}\right\} \rightarrow\left\{\overline{e_{1}}, \ldots, \overline{e_{n-1}}, k z_{1}\right\}$, with $k \in \mathbb{F}^{*}$, of $L_{1}$. Thus the isomorphism between $L_{1}$ and $L_{2}$ is given by the linear map

$$
\varphi\left(\overline{e_{i}}\right)=e_{i}^{\prime}, \forall i=1, \ldots, n-1, \varphi\left(k z_{1}\right)=z_{2},
$$

where $\left\{e_{1}^{\prime}, \ldots, e_{n-1}^{\prime}, z_{2}\right\}$ is a basis of $L_{2}$.

We observe that, if $X \in \mathrm{GL}_{n}(\mathbb{F})$, then the matrix

$$
\left(\begin{array}{cc}
X & 0 \\
0 & \left(X^{-1}\right)^{t}
\end{array}\right)
$$

induces a change of basis that transforms the canonical pairs (1) and (2) respectively in

$$
\left(\left(\begin{array}{cc}
0 & I_{n} \\
-I_{n} & 0
\end{array}\right),\left(\begin{array}{cc}
0 & X A X^{-1} \\
\left(X A X^{-1}\right)^{t} & 0
\end{array}\right)\right),\left(\left(\begin{array}{cc}
0 & X A X^{-1} \\
-\left(X A X^{-1}\right)^{t} & 0
\end{array}\right),\left(\begin{array}{cc}
0 & I_{n} \\
I_{n} & 0
\end{array}\right)\right)
$$

so we can always reduce $A$ in its rational canonical form. Thus, with the assumption that $L$ is indecomposable, if $A \in \mathrm{M}_{n}(\mathbb{F})$ is not the zero matrix, then $A$ is the companion matrix of a power of a monic irreducible polynomial $f(x) \in \mathbb{F}[x]$ (see $[10]$ ).

Moreover, if $A$ is not a singular matrix, then the second canonical pair is equivalent to the first one, in fact

$$
\begin{aligned}
& \left(\begin{array}{cc}
A^{-1} & 0 \\
0 & I_{n}
\end{array}\right)\left(\begin{array}{cc}
0 & A \\
-A^{t} & 0
\end{array}\right)\left(\begin{array}{cc}
A^{-1} & 0 \\
0 & I_{n}
\end{array}\right)^{t}=\left(\begin{array}{cc}
0 & I_{n} \\
-I_{n} & 0
\end{array}\right) \\
& \left(\begin{array}{cc}
A^{-1} & 0 \\
0 & I_{n}
\end{array}\right)\left(\begin{array}{cc}
0 & I_{n} \\
I_{n} & 0
\end{array}\right)\left(\begin{array}{cc}
A^{-1} & 0 \\
0 & I_{n}
\end{array}\right)^{t}=\left(\begin{array}{cc}
0 & A^{-1} \\
\left(A^{-1}\right)^{t} & 0
\end{array}\right) .
\end{aligned}
$$

Otherwise, we can represent a singular matrix as an $n \times n$ Jordan block of eigenvalue zero and we have a unique Kronecker module of type (2) up to isomorphisms.

Definition 2.3. Let $f(x) \in \mathbb{F}[x]$ be a monic irreducible polynomial. Let $k \in \mathbb{N}$ and let $A$ be the companion matrix of $f(x)^{k}$. We define the Heisenberg Leibniz algebra $\mathfrak{l}_{2 n+1}^{A}$ as the $(2 n+1)$-dimensional indecomposable Leibniz algebra with associated Kronecker module of type (1).

In general, if $A=\left(a_{i j}\right) \in \mathrm{M}_{n}(\mathbb{F})$ and if we fix a basis $\left\{e_{1}, \ldots, e_{n}, f_{1}, \ldots, f_{n}, h\right\}$ of $\mathfrak{r}_{2 n+1}^{A}$, then the non-trivial commutators are

$$
\left[e_{i}, f_{j}\right]=\left(\delta_{i j}+a_{i j}\right) h,\left[f_{j}, e_{i}\right]=\left(-\delta_{i j}+a_{i j}\right) h, \forall i, j=1, \ldots, n,
$$

so we can associate with $\mathfrak{l}_{2 n+1}^{A}$ the following structure matrix

$$
\left(\begin{array}{c|c|c} 
& & 0 \\
0 & I_{n}+A & \vdots \\
& & 0 \\
\hline & & 0 \\
-I_{n}+A^{t} & 0 & \vdots \\
& & 0 \\
\hline 0 \cdots 0 & 0 \cdots 0 & 0
\end{array}\right)
$$

Notice that, if $\left(a_{i j}\right)$ is the zero matrix, then we obtain the classical Heisenberg algebra $\mathfrak{h}_{2 n+1}$. 
Definition 2.4. Let $n \in \mathbb{N}$ and let $A$ be the companion matrix of the polynomial $x^{n}$. We define the Kronecker Leibniz algebra $\mathfrak{k}_{n}$ as the $(2 n+1)$-dimensional indecomposable Leibniz algebra with associated Kronecker module of type (2).

Definition 2.5. We define the Dieudonné Leibniz algebra $\mathfrak{d}_{n}$ is the $(2 n+2)$-dimensional Leibniz algebra with associated Kronecker module of type (3).

We observe that the Kronecker Leibniz algebra and the Dieudonné Leibniz algebra are not Lie algebras and they are unique up to isomorphisms.

\section{Complex and Real Heisenberg Leibniz algebras}

Now we want to describe in details the indecomposable Heisenberg Leibniz algebras in the case the field $\mathbb{F}$ is $\mathbb{C}$ or $\mathbb{R}$.

\subsection{The case $\mathbb{F}=\mathbb{C}$}

Let $k \in \mathbb{N}$ and let $f(x)=x-a \in \mathbb{C}[x]$. Then the companion matrix of $f(x)^{k}$ is

$$
A=\left(\begin{array}{ccccc}
0 & \cdots & \cdots & 0 & -c_{k} \\
1 & \ddots & & \vdots & -c_{k-1} \\
0 & 1 & \ddots & \vdots & \vdots \\
\vdots & \ddots & \ddots & 0 & \vdots \\
0 & \cdots & 0 & 1 & -c_{1}
\end{array}\right) \in \mathrm{M}_{k}(\mathbb{C})
$$

where $c_{j}=\left(\begin{array}{c}k \\ j-1\end{array}\right)(-a)^{k-j+1}$, for every $j=1, \ldots, k$. In this case, however, it is more convenient to use the Jordan canonical form. Indeed, it is well known that the matrix $A$ is similar to the $k \times k$ Jordan block of eigenvalue $a$

$$
J_{a}=\left(\begin{array}{ccccc}
a & 0 & 0 & \cdots & 0 \\
1 & \ddots & \ddots & & \vdots \\
\vdots & \ddots & \ddots & \ddots & 0 \\
\vdots & & \ddots & \ddots & 0 \\
0 & \cdots & \cdots & 1 & a
\end{array}\right)
$$

Thus $\mathfrak{l}_{2 k+1}^{A} \cong \mathfrak{l}_{2 k+1}^{J_{a}}$ and the Leibniz bracket is given by

$$
\begin{aligned}
& {\left[e_{1}, f_{j}\right]=\delta_{1, j}(1+a) z} \\
& {\left[e_{i}, f_{j}\right]=\left(\delta_{i, j}(1+a)+\delta_{i-1, j}\right) z, \forall i=2, \ldots, k ;} \\
& {\left[f_{j}, e_{i}\right]=\left(\delta_{i, j}(-1+a)+\delta_{i, j+1}\right) z, \forall j=1, \ldots, k-1} \\
& {\left[f_{k}, e_{i}\right]=\delta_{i, k}(-1+a) z,}
\end{aligned}
$$

where $\left\{e_{1}, \ldots, e_{n}, f_{1}, \ldots, f_{n}, h\right\}$ is a basis of $\mathfrak{l}_{2 k+1}^{J_{a}}$.

Proposition 3.1. Let $a \in \mathbb{C}$. The Heinseberg-Leibniz algebras $\mathfrak{l}_{2 k+1}^{J_{a}}$ and $\mathfrak{l}_{2 k+1}^{J_{-a}}$ are isomorphic.

Proof. The algebras $\mathfrak{l}_{2 k+1}^{J_{a}}$ and $\mathfrak{l}_{2 k+1}^{-J_{a}^{t}}$ are isomorphic via the linear map $\varphi$ defined by

$$
\varphi\left(e_{i}\right)=f_{i}^{\prime}, \varphi\left(f_{i}\right)=e_{i}^{\prime}, \varphi(h)=-h^{\prime}, \forall i=1, \cdots, n
$$

where $\left\{e_{1}, \cdots, e_{n}, f_{1}, \cdots, f_{n}, h\right\}$ and $\left\{e_{1}^{\prime}, \cdots, e_{n}^{\prime}, f_{1}^{\prime}, \cdots, f_{n}^{\prime}, h^{\prime}\right\}$ are bases of $\mathfrak{l}_{2 k+1}^{J_{a}}$ and $\mathfrak{r}_{2 k+1}^{-J_{a}^{t}}$ respectively. Moreover the matrix $-J_{a}^{t}$ is similar to the $n \times n$ Jordan block $J_{-a}$. Thus $\mathfrak{l}_{2 k+1}^{J_{a}} \cong \mathfrak{l}_{2 k+1}^{J_{-a}}$. 
When $k=1$ the converse result is also true.

Proposition 3.2. Let $a, a^{\prime} \in \mathbb{C}$. The Heisenberg Leibniz algebras $\mathfrak{l}_{3}^{a}$ and $\mathfrak{l}_{3}^{a^{\prime}}$ are isomorphic if and only if $a^{\prime}= \pm a$.

Proof. It is easy to check that the matrix

$$
\left(\begin{array}{ccc}
0 & 1 & 0 \\
1 & 0 & 0 \\
0 & 0 & -1
\end{array}\right)
$$

defines a Leibniz algebras isomorphism between $\mathfrak{l}_{3}^{a}$ and $\mathfrak{l}_{3}^{-a}$. Conversely, let $\varphi: \mathfrak{l}_{3}^{a} \rightarrow \mathfrak{l}_{3}^{a^{\prime}}$ be a Leibniz algebra isomorphism defined by

$$
\varphi(x)=\alpha x^{\prime}+\beta y^{\prime}+\gamma z^{\prime}, \varphi(y)=\alpha^{\prime} x^{\prime}+\beta^{\prime} y^{\prime}+\gamma^{\prime} z^{\prime}, \varphi(z)=k z^{\prime},
$$

where $\{x, y, z\}$ and $\left\{x^{\prime}, y^{\prime}, z^{\prime}\right\}$ are basis of $\mathfrak{l}_{3}^{a}$ and $\mathfrak{l}_{3}^{a^{\prime}}$ respectively. Thus

$$
\begin{gathered}
0=\varphi([x, x])=[\varphi(x), \varphi(x)]=\alpha \beta\left(1+a^{\prime}-1+a^{\prime}\right) z^{\prime}=2 \alpha \beta a^{\prime} z^{\prime}, \\
0=\varphi([y, y])=[\varphi(y), \varphi(y)]=\alpha^{\prime} \beta^{\prime}\left(1+a^{\prime}-1+a^{\prime}\right) z^{\prime}=2 \alpha^{\prime} \beta^{\prime} a^{\prime} z^{\prime}, \\
k(1+a) z^{\prime}=\varphi([x, y])=[\varphi(x), \varphi(y)]=\left(\alpha^{\prime} \beta\left(1+a^{\prime}\right)+\alpha \beta^{\prime}\left(-1+a^{\prime}\right)\right) z^{\prime}, \\
k(-1+a) z^{\prime}=\varphi([y, x])=[\varphi(y), \varphi(x)]=\left(\alpha \beta^{\prime}\left(1+a^{\prime}\right)+\alpha^{\prime} \beta\left(-1+a^{\prime}\right)\right) z^{\prime} .
\end{gathered}
$$

We have that $\left(\alpha^{\prime}, \beta\right)=(0,0)$ or $\left(\alpha, \beta^{\prime}\right)=(0,0)$. In the first case $\varphi$ is the identity function and $a=a^{\prime}$. In the second case $\varphi$ is defined by $\varphi(x)=y^{\prime}, \varphi(y)=x^{\prime}$ and $\varphi(z)=-z^{\prime}$, thus $a^{\prime}=-a$.

\subsection{The case $\mathbb{F}=\mathbb{R}$}

Irreducible polynomials in $\mathbb{R}[x]$ have degree one or two. Let $f(x) \in \mathbb{R}[x]$ be an irreducible monic polynomial. If $f(x)=x-a$, then we obtain the same results of the previous case. So we suppose that $f(x)=x^{2}+b x+c$, with $b^{2}-4 c<0$.

Let $z=\alpha+i \beta \in \mathbb{C}$ be a root of $f(x)$. Then $f(x)=(x-z)(x-\bar{z})$ and the companion matrix $A$ of $f(x)^{k}$ in similar to the $2 k \times 2 k$ real block matrix

$$
J_{R}=\left(\begin{array}{cccc}
R & 0 & \cdots & 0 \\
I_{2} & R & \cdots & 0 \\
\vdots & \ddots & \ddots & \vdots \\
0 & \cdots & I_{2} & R
\end{array}\right)
$$

where

$$
R=R_{\alpha, \beta}=\left(\begin{array}{cc}
\alpha & \beta \\
-\beta & \alpha
\end{array}\right)
$$

is the realification of the complex number $z$. Thus $\mathfrak{l}_{4 k+1}^{A} \cong \mathfrak{l}_{4 k+1}^{J_{R}}$ and the structure matrix is given by

$$
\left(\begin{array}{c|c|c}
0 & I_{n}+J_{R} & \vdots \\
0 & 0 \\
\hline-I_{n}+J_{R}^{t} & 0 & \vdots \\
\hline 0 \cdots 0 & 0 \cdots 0 & 0
\end{array}\right)
$$


In the case that $k=1$, the real Heisenberg Leibniz algebra $\mathfrak{l}_{5}^{R}$ is the realification of the complex algebra $\mathfrak{l}_{3}^{z}$. Thus we can conclude that

Proposition 3.3. Let $f(x), g(x) \in \mathbb{R}[x]$ be two irreducible monic polynomials of degree two and let $z, z^{\prime} \in \mathbb{C}$ be roots of $f(x)$ and $g(x)$ respectively. Let $R, R^{\prime} \in \mathrm{M}_{2}(\mathbb{R})$ be the realification of the complex numbers $z$ and $z^{\prime}$. Then $\mathfrak{l}_{5}^{R} \cong \mathfrak{l}_{5}^{R^{\prime}}$ if and only if $R^{\prime}= \pm R$.

Proof. The algebras $\mathfrak{l}_{5}^{R}$ and $\mathfrak{l}_{5}^{R^{\prime}}$ are the realification of the complex Heisenberg Leibniz algebras $\mathfrak{l}_{3}^{z}$ and $\mathfrak{l}_{3}^{z^{\prime}}$ respectively. From Proposition 3.2 we know that $\mathfrak{l}_{3}^{z} \cong \mathfrak{l}_{3}^{z^{\prime}}$ if and only if $z= \pm z^{\prime}$. Moreover, these are $\mathbb{R}$-linear isomorphisms because the matrix associated with the isomorphism $\varphi: \mathfrak{l}_{3}^{z} \leftrightarrows \mathfrak{l}_{3}^{-z}$ is the rotation

$$
\left(\begin{array}{ccc}
0 & 1 & 0 \\
1 & 0 & 0 \\
0 & 0 & -1
\end{array}\right) \in \mathrm{SO}(3)
$$

Thus $\mathfrak{l}_{5}^{R} \cong \mathfrak{l}_{5}^{R^{\prime}}$ if and only if $R= \pm R^{\prime}$.

\section{Global integration of two-step nilpotent Leibniz algebras}

In the case of the correspondence between a Lie group $G$ and its Lie algebra $\mathfrak{g}=T_{1} G$, the bracket

$$
[x, y]=\operatorname{ad}_{x}(y),
$$

where ad $: \mathfrak{g} \rightarrow \mathfrak{g l}(\mathfrak{g})$, is the differential of the adjoint representation Ad $: G \rightarrow \operatorname{GL}(\mathfrak{g})$, which in turn is the differential of the conjugation map $\gamma: x \mapsto \gamma_{x}$, where $\gamma_{x}(y):=x \triangleright y$ and $x \triangleright y=x y x^{-1}$, for every $x, y \in G$. In the case of Leibniz algebras, there is no hope, as we will see, of finding such a map Ad, but it is still possible to define an algebraic structure $(X, \triangleright)$, called a rack, whose operation, differentiated twice, defines on $T_{1} X$ a Leibniz algebra structure.

From now on, unless explicitly stated, the underlying field of any vector space will be the real numbers.

Definition 4.1. A (left) rack is a set $X$ with a binary operation $\triangleright: X \times X \rightarrow X$ which is (left) autodistributive, that is, for all $x, y, z \in X, x \triangleright(y \triangleright z)=(x \triangleright y) \triangleright(x \triangleright z)$ and such that $x \triangleright-: X \times X \rightarrow X$ is a bijection for all $x \in X$. A rack is said to be pointed if there exists an element $1 \in X$, called the unit, such that $1 \triangleright x=x$ and $x \triangleright 1=1$ for all $x \in X$. A rack $(X, \triangleright)$ is a quandle if $x \triangleright x=x$, for every $x \in X$ (i.e. $\triangleright$ is idempotent).

A pointed rack homomorphism is a map $f: X \rightarrow Y$ such that $f(x \triangleright y)=f(x) \triangleright f(y)$, for all $x, y \in X$ and such that $f\left(1_{X}\right)=1_{Y}$.

Every group endowed of the conjugation is a pointed rack, so it is defined a functor Conj : $\mathbf{G r p} \rightarrow \mathbf{R a c k}$, between the category Grp of groups and the category Rack of racks.

This functor has a left adjoint As : Rack $\rightarrow$ Grp defined by

$$
\operatorname{As}(X)=\mathrm{F}(X) / \overline{\left\langle\left\{x y x^{-1}\left(x \triangleright y^{-1}\right) \mid x y \in X\right\}\right\rangle},
$$

where $\mathrm{F}(X)$ is the free group generated by $X$.

Definition 4.2. A Lie rack is a pointed rack $(X, \triangleright, 1)$ such that $X$ is a smooth manifold, $\triangleright$ is a smooth map and such that for all $x \in X x \triangleright-$ is a diffeomorphism.

In [11] M.K. Kynion shows that the tangent space at the unit element of a Lie rack $(X, \triangleright)$, endowed with the bracket

$$
[x, y]=\operatorname{ad}_{x}(y),
$$

where ad $: T_{1} X \rightarrow \mathfrak{g l}\left(T_{1} X\right)$ is the differential of the map $\Phi: X \mapsto \operatorname{GL}\left(T_{1} X\right)$, where $\Phi(x)=T_{1} \phi(x)$ and $\phi(x)=x \triangleright-$, for every $x \in X$. Summarizing we have 
Proposition 4.3. [11] If $X$ be a Lie rack, then the above bracket $[x, y]=\operatorname{ad}_{x}(y)$ defines on $T_{1} X$ a Leibniz algebra structure.

The converse problem, that is to find a manifold endowed of an algebraic operation such that the tangent space at a distinguished point, endowed of the differential of such operation, gives a Leibniz algebra isomorphic to the given one, is known as the coquecigrue problem. In [11] M. Kynion solves the coquecigrue problem for the class of split Leibniz algebras, that are Leibniz algebras $\mathfrak{g}$ with a bilateral ideal $\operatorname{Leib}(\mathfrak{g}) \subseteq \mathfrak{a} \subseteq \mathrm{Z}_{l}(\mathfrak{g})$ and with a Lie subalgebra $\mathfrak{h} \subseteq \mathfrak{g}$ such that $\mathfrak{g}=\mathfrak{h} \oplus \mathfrak{a}$ (as a direct sum of vector spaces) and

$$
[(x, a),(y, b)]=\left([x, y], \rho_{x}(b)\right), \quad \forall(x, a),(y, b) \in \mathfrak{h} \oplus \mathfrak{a}
$$

where $\rho: \mathfrak{h} \times \mathfrak{a} \rightarrow \mathfrak{a}$ is the action on the $\mathfrak{h}$-module $\mathfrak{a}$. More precisely, we have the following.

Proposition 4.4. [11] Let $\mathfrak{g}$ be a split Leibniz algebra. Then there exists a Lie rack $X$ such that $T_{1} X$ is isomorphic to $\mathfrak{g}$.

More recently S. Covez in [12] gives a solution to this problem which in general is only local: he shows how to integrate every Leibniz algebra into a local Lie rack. The central point of his result is to see every Leibniz algebra $\mathfrak{g}$ as a central extension of his left center $\mathrm{Z}_{l}(\mathfrak{g})$ and to integrate explicitly the corresponding Leibniz algebra 2-cocycle into a Lie local rack 2-cocycle. However M. Bordemann and F. Wagemann (see [13]) and J. Mostovoy (see [14]) give independently two different answers to the general coquecigrue problem: Bordemann and Wagemann's solution is not functorial; Mostovoy's solution is global but does not generalize the classical Lie solution. The general coquecigrue problem is still open.

The aim of this section is to use the Leibniz algebras - Lie local racks correspondence proposed by S. Covez to show that the integration of the two-step nilpotent Leibniz algebras is global.

In [12] S. Covez gives the definition of smooth rack modules, rack cohomology and cohomolgy theory for Leibniz algebras. In particular, for $X$ a Lie rack and $A$ a smooth $X$-module, he defines a cochain complex $\left\{\mathrm{CR}^{n}(X, A), d_{R}^{n}\right\}_{n \in \mathbb{N}}$ by setting

$\mathrm{CR}^{n}(X, A)=\left\{f: X^{n} \rightarrow A \mid f\left(x_{1}, \ldots, 1, \ldots, x_{n}\right)=0, f\right.$ is smooth in a neighborhood of $\left.(1, \ldots, 1) \in X^{n}\right\}$

and $d_{R}^{n}$ is the differential operator. Moreover, for $\mathfrak{g}$ a left Leibniz algebra and $M$ a $\mathfrak{g}$-module, he defines a cochain complex $\left\{\mathrm{CL}^{n}(\mathfrak{g}, M), d L^{n}\right\}_{n \in \mathbb{N}}$ by setting

$$
\mathrm{CL}^{n}(\mathfrak{g}, M)=\operatorname{Hom}\left(\mathfrak{g}^{\otimes n}, M\right)
$$

and $d L^{n}$ is the differential operator.

Given a left Leibniz algebra $\mathfrak{g}$, there are several ways to see $\mathfrak{g}$ as a central extension of a Lie subalgebra $\mathfrak{g}_{0} \subseteq \mathfrak{g l}(V)$ by a $\mathfrak{g}_{0}-$ module $\mathfrak{a}$. For example we can take $\mathfrak{a}=\mathrm{Z}_{l}(\mathfrak{g})$ and $\mathfrak{g}_{0}=\mathfrak{g} / \mathrm{Z}_{l}(\mathfrak{g})$. Thus we can associate with $\mathfrak{g}$ a short exact sequence

$$
0 \rightarrow \mathrm{Z}_{l}(\mathfrak{g}) \hookrightarrow \mathfrak{g} \rightarrow \mathfrak{g}_{0} \rightarrow 0 .
$$

in the category LeibAlg. $\mathrm{Z}_{l}(\mathfrak{g})$ is a $\mathfrak{g}_{0}$-module (in the sense of Lie algebras), so there is a Leibniz algebras 2 -cocycle $\omega \in \mathrm{ZL}^{2}\left(\mathfrak{g}_{0}, \mathrm{Z}_{l}(\mathfrak{g})\right)$ such that $\mathfrak{g}=\mathfrak{g}_{0} \oplus_{\omega} \mathrm{Z}_{l}(\mathfrak{g})$. The Leibniz bracket in $\mathfrak{g}$ can be written as follows

$$
[(x, a),(y, b)]=\left([x, y]_{\mathfrak{g}_{0}}, \rho_{x}(b)+\omega(x, y)\right),
$$

where $\rho: \mathfrak{g}_{0} \times \mathrm{Z}_{l}(\mathfrak{g}) \rightarrow \mathrm{Z}_{l}(\mathfrak{g})$ is the action induced by the $\mathfrak{g}_{0}$-module structure of $\mathrm{Z}_{l}(\mathfrak{g})$.

Theorem 4.5. [12] Every Leibniz algebra $\mathfrak{g}=\mathfrak{g}_{0} \oplus_{\omega} \mathfrak{a}$ can be integrated into a local Lie rack of the form

$$
G_{0} \times_{f} \mathfrak{a}
$$

with operation defined by

$$
(g, a) \triangleright(h, b)=\left(g h g^{-1}, \phi_{g}(b)+f(g, h)\right)
$$


and unit $(1,0)$, where $G_{0}$ is a Lie group such that $\operatorname{Lie}\left(G_{0}\right)=\mathfrak{g}_{0}, \phi$ is the exponentiation of the action $\rho$, $f: G_{0} \times G_{0} \rightarrow \mathfrak{a}$ is the Lie local racks 2 - cocycle defined by

$$
f(g, h)=\int_{\gamma_{h}}\left(\int_{\gamma_{g}} \tau^{2}(\omega)^{e q}\right)^{e q}, \forall g, h \in G_{0}
$$

and $\tau^{2}(\omega) \in \mathrm{ZL}^{1}\left(\mathfrak{g}_{0}, \operatorname{Hom}\left(\mathfrak{g}_{0}, \mathfrak{a}\right)\right)$ is defined by $\tau^{2}(\omega)(x)(y)=\omega(x, y)$, for all $x, y \in \mathfrak{g}_{0}$.

We finally can answer the question whether a Lie rack integrating a Leibniz algebra can be the quandle $\operatorname{Conj}(G)$, for a suitable Lie group $G$. The answer is no in general, as the following theorem shows.

Theorem 4.6. Let $R$ be a Lie rack integrating a Leibniz algebra $\mathfrak{g} . R$ is a quandle if and only if $\mathfrak{g}$ is a Lie algebra. In particular $R=\operatorname{Conj}(G)$, where $\operatorname{Lie}(G)=\mathfrak{g}$.

Proof. If $\mathfrak{g}$ is a Lie algebra, then it is clear that $R=\operatorname{Conj}(G)$, where $\operatorname{Lie}(G)=\mathfrak{g}$. Conversely, we suppose that $R$ is a Lie quandle. Again we can write $\mathfrak{g}=\mathfrak{g}_{0} \oplus_{\omega} \mathrm{Z}_{l}(\mathfrak{g})$, thus $R$ is of the form $G_{0} \times{ }_{f} \mathrm{Z}_{l}(\mathfrak{g})$, with multiplication

$$
(g, a) \triangleright(h, b)=\left(g h g^{-1}, \phi_{g}(b)+f(g, h)\right),
$$

where $f$ is the Lie racks 2 -cocycle integrating $\omega$. To prove that $\mathfrak{g}$ is a Lie algebra, we have to show that $[(x, a),(x, a)]=(0,0)$, for all $(x, a) \in \mathfrak{g}$.

The condition $(g, a) \triangleright(g, a)=(g, a)$ implies that $f(g, g)=0$, for all $g \in G_{0}$, and then $\phi_{g}(a)=a$, for all $a \in Z_{l}(\mathfrak{g})$. Indeed the action $\rho$ of $\mathfrak{g}_{0}$ on $\mathrm{Z}_{l}(\mathfrak{g})$ is trivial and $\omega(x, x)=0$ for all $x \in \mathfrak{g}_{0}$. Finally $R=\operatorname{Conj}(G)$, where $G=G_{0} \times_{F} \mathrm{Z}_{l}(\mathfrak{g})$ is the Lie group with operation

$$
(g, a)(h, b)=(g h, a+b+F(g, h)),
$$

and $F: G_{0} \times G_{0} \rightarrow \mathrm{Z}_{l}(\mathfrak{g})$ is a Lie group 2-cocycle such that

$$
f(g, h)=F(g, h)-F\left(g, g^{-1}\right)+F\left(g h, g^{-1}\right) \quad \forall g, h \in G_{0} .
$$

In fact with this condition we have that

$$
(g, a) \triangleright(h, b)=(g, a)(h, b)(g, a)^{-1} \quad \forall(g, a),(h, b) \in G_{0} \times \mathrm{Z}_{l}(\mathfrak{g})
$$

and the Lie algebra of the Lie group $G$ is clearly $\mathfrak{g}$.

Now we will claim a result about the integration of two-step nilpotent Leibniz algebras. We will show that, for this class of Leibniz algebras, the integration proposed by Covez is global. Moreover, a Lie rack integrating $\mathfrak{g}$ can be defined without integrating the Leibniz algebras 2 -cocycle associated with $\mathfrak{g}$.

Theorem 4.7. Let $(\mathfrak{g},[-,-])$ be a two-step nilpotent Leibniz algebra and let $\omega: \mathfrak{g}_{0} \times \mathfrak{g}_{0} \rightarrow[\mathfrak{g}, \mathfrak{g}]$, where $\mathfrak{g}_{0}=\mathfrak{g} /[\mathfrak{g}, \mathfrak{g}]$, be the Leibniz algebras 2 -cocycle associated with the short exact sequence

$$
0 \rightarrow[\mathfrak{g}, \mathfrak{g}] \hookrightarrow \mathfrak{g} \rightarrow \mathfrak{g}_{0} \rightarrow 0
$$

Then the multiplication

$$
(x, a) \triangleright(y, b)=(y, b+\omega(x, y)), \quad \forall(x, a),(y, b) \in \mathfrak{g}_{0} \times[\mathfrak{g}, \mathfrak{g}]
$$

defines a Lie global rack structure on $\mathfrak{g}_{0} \times[\mathfrak{g}, \mathfrak{g}]$, such that $T_{(0,0)}\left(\mathfrak{g}_{0} \times \omega[\mathfrak{g}, \mathfrak{g}], \triangleright\right)$ is a Leibniz algebra isomorphic to $\mathfrak{g}$.

Proof. We have $[\mathfrak{g}, \mathfrak{g}] \subseteq Z(\mathfrak{g})$, so we can see $\mathfrak{g}$ as a central extension of $[\mathfrak{g}, \mathfrak{g}]$ by the quotient $\mathfrak{g}_{0}=\mathfrak{g} /[\mathfrak{g}, \mathfrak{g}]$ via a Leibniz algebras 2 -cocycle $\omega \in \mathrm{ZL}^{2}\left(\mathfrak{g}_{0},[\mathfrak{g}, \mathfrak{g}]\right)$. Thus $\mathfrak{g}=\mathfrak{g}_{0} \oplus_{\omega}[\mathfrak{g}, \mathfrak{g}]$ with bracket

$$
[(x, a),(y, b)]=(0, \omega(x, y))
$$


In fact the condition $[\mathfrak{g}, \mathfrak{g}] \subseteq \mathrm{Z}_{l}(\mathfrak{g}) \cap \mathrm{Z}_{r}(\mathfrak{g})$ implies that the action of $\mathfrak{g}_{0}$ on $[\mathfrak{g}, \mathfrak{g}]$ is trivial. Moreover $\mathfrak{g}_{0}$ is an abelian Lie algebra, thus a Lie group integrating $\mathfrak{g}_{0}$ is $G_{0}=\mathfrak{g}_{0}$. Then we can define a Lie rack structure on the cartesian product $\mathfrak{g}_{0} \times[\mathfrak{g}, \mathfrak{g}]$ by setting

$$
(x, a) \triangleright(y, b)=(y, b+\omega(x, y)) \quad \forall(x, a),(y, b) \in \mathfrak{g}_{0} \times[\mathfrak{g}, \mathfrak{g}],
$$

with unit element $(0,0)$. Finally the tangent space $T_{(0,0)}\left(\mathfrak{g}_{0} \times[\mathfrak{g}, \mathfrak{g}]\right)$ has a Leibniz algebra structure isomorphic to $\mathfrak{g}$. In fact

$$
\left.\frac{\partial^{2}}{\partial s \partial t}\right|_{s, t=0}(s x, s a) \triangleright(t y, t b)=\left.\frac{\partial^{2}}{\partial s \partial t}\right|_{s, t=0}(t y, t b+\omega(s x, t y))=(0, \omega(x, y))=[(x, a),(y, b)] .
$$

Remark 4.8. The effective strategy in the proof of Theorem 4.7 was to choose $G_{0}=\mathfrak{g}_{0}$ as a Lie group integrating the abelian Lie algebra $\mathfrak{g}_{0}$. If we change the Lie group $G_{0}$, then the integration may not be global, as the following example illustrates.

Example 4.9. Let $a \in \mathbb{R}$ and let $\mathfrak{g}=\mathfrak{l}_{3}^{a}$ be the three-dimensional Heinseberg Lebiniz algebra. Then $[\mathfrak{g}, \mathfrak{g}]=$ $\mathrm{Z}(\mathfrak{g}) \cong \mathbb{R}$ and we can see $\mathfrak{g}$ as a central extension of the abelian Lie algebra $\mathfrak{g}_{0}=\mathfrak{g} /[\mathfrak{g}, \mathfrak{g}] \cong \mathbb{R}^{2}$ by $\mathbb{R}$. The corresponding Leibniz algebras 2-cocycle is

$$
\omega\left((x, y),\left(x^{\prime}, y^{\prime}\right)\right)=(1+a) x y^{\prime}+(-1+a) x^{\prime} y .
$$

Now we can choose

$$
G_{0}=\mathrm{SO}(2) \times \mathrm{SO}(2) \cong\left\{\left(e^{i x}, e^{i y}\right) \mid x, y \in \mathbb{R}\right\}
$$

as a Lie group integrating $\mathfrak{g}_{0}$. In this case a Lie local rack integrating $\mathfrak{g}$ is $\left(G_{0} \times \mathrm{SO}(2)\right.$, $\left.\triangleright\right)$ with multiplication

$$
\left(e^{i x}, e^{i y}, e^{i z}\right) \triangleright\left(e^{i x^{\prime}}, e^{i y^{\prime}}, e^{i z^{\prime}}\right)=\left(e^{i x^{\prime}}, e^{i y^{\prime}}, e^{i\left(z^{\prime}+\omega\left(\left(\log \left(e^{i x}\right), \log \left(e^{i y}\right)\right),\left(\log \left(e^{i x^{\prime}}\right), \log \left(e^{i y^{\prime}}\right)\right)\right)\right.}\right),
$$

that is defined only for $(x, y),\left(x^{\prime}, y^{\prime}\right) \in[0,2 \pi[\times[0,2 \pi[$, where we choose $[0,2 \pi[$ as the domain of the principal value of the function log. Thus the integration is not global.

In order to show that Theorem 4.7 provides an effective tool for the construction of a global rack integrating a Leibniz algebra $\mathfrak{g}$ with $[\mathfrak{g}, \mathfrak{g}] \subseteq \mathrm{Z}(\mathfrak{g})$, we can reformulate an example proposed by S. Covez in [12].

Example 4.10. Let $\mathfrak{g}=\left(\mathbb{R}^{4},[-,-]\right)$ with basis $\left\{e_{1}, e_{2}, e_{3}, e_{4}\right\}$ and nonzero brackets

$$
\begin{gathered}
{\left[e_{1}, e_{1}\right]=\left[e_{1}, e_{2}\right]=\left[e_{2}, e_{2}\right]=\left[e_{3}, e_{3}\right]=e_{4},} \\
{\left[e_{2}, e_{1}\right]=-e_{4} .}
\end{gathered}
$$

It is easy to see that $\mathfrak{g}$ is a left Leibniz algebra with $[\mathfrak{g}, \mathfrak{g}]=\mathrm{Z}(\mathfrak{g})=\mathbb{R} e_{4}$. We have that $\mathfrak{g}=\mathfrak{g}_{0} \oplus_{\omega} \mathbb{R} e_{4}$, where $\mathfrak{g}_{0} \cong \operatorname{Span}_{\mathbb{R}}\left\{e_{1}, e_{2}, e_{3}\right\}$, and the Leibniz 2-cocycle is given by

$$
\omega(x, y)=\left[\left(x_{1}, x_{2}, x_{3}, 0\right),\left(y_{1}, y_{2}, y_{3}, 0\right)\right]=\left(0,0,0, x_{1} y_{1}+x_{1} y_{2}-x_{2} y_{1}+x_{2} y_{2}+x_{3} y_{3}\right) .
$$

Thus, by Theorem 4.7, a Lie global rack integrating $\mathfrak{g}$ is $\left(\mathfrak{g}_{0} \times_{\omega} \mathbb{R}_{4}, \triangleright\right)$ with multiplication given by

$$
\left(x_{1}, x_{2}, x_{3}, x_{4}\right) \triangleright\left(y_{1}, y_{2}, y_{3}, y_{4}\right)=\left(y_{1}, y_{2}, y_{3}, y_{4}+\omega(x, y)\right) .
$$

Now we can globally integrate all the indecomposable nilpotent real Leibniz algebras with one-dimensional commutator ideal classified in the previous sections. For the Heisenberg Leibniz algebras, we will obtain Lie racks that are "perturbations" of the conjugation of the Heisenberg Lie group $H_{2 n+1}$. From now on we suppose that $A \in \mathrm{M}_{n}(\mathbb{R})$ is the companion matrix of the power of an irreducible monic polynomial $f(x) \in \mathbb{R}[x]$. Thus $A$ is a $n \times n$ Jordan block of eigenvalue $a \in \mathbb{R}$ or $A=J_{R}$, where $R \in \mathrm{M}_{2}(\mathbb{C})$ is the realification of some complex number $z=\alpha+i \beta$. 
Example 4.11. Let $\mathfrak{g}=\mathfrak{l}_{2 n+1}^{A}$ and let $\left\{e_{1}, \ldots, e_{n}, f_{1}, \ldots, f_{n}, h\right\}$ be a basis of $\mathfrak{g}$. Then $[\mathfrak{g}, \mathfrak{g}]=\mathbb{R} h \subseteq \mathrm{Z}(\mathfrak{g})$, thus we can use Theorem 4.7 to find the Lie global rack integrating $\mathfrak{g}$. The Leibniz bracket of $\mathfrak{g}$ is given by

$\left[\left(x_{1}, \ldots, x_{n}, y_{1}, \ldots, y_{n}, z\right),\left(x_{1}^{\prime}, \ldots, x_{n}^{\prime}, y_{1}^{\prime}, \ldots, y_{n}^{\prime}, z^{\prime}\right)\right]=\left(0, \ldots, 0, \sum_{i, j=1}^{n}\left(\delta_{i j}+a_{i j}\right) x_{i} y_{j}^{\prime}+\sum_{i, j=1}^{n}\left(-\delta_{i j}+a_{i j}\right) x_{i}^{\prime} y_{j}\right)$,

so we obtain a Lie rack $R_{2 n+1}^{A}=\left(\mathfrak{g}_{0} \times_{f} \mathbb{R} h, \triangleright\right)$ with multiplication

$$
\begin{aligned}
\left(x_{1}, \ldots, x_{n}, y_{1}, \ldots, y_{n}, z\right) & \triangleright\left(x_{1}^{\prime}, \ldots, x_{n}^{\prime}, y_{1}^{\prime}, \ldots, y_{n}^{\prime}, z^{\prime}\right)= \\
& =\left(x_{1}^{\prime}, \ldots, x_{n}^{\prime}, y_{1}^{\prime}, \ldots, y_{n}^{\prime}, z^{\prime}+\sum_{i, j=1}^{n}\left[\left(\delta_{i j}+a_{i j}\right) x_{i} y_{j}^{\prime}+\left(-\delta_{i j}+a_{i j}\right) x_{i}^{\prime} y_{j}\right]\right)
\end{aligned}
$$

and $T_{(0,0)} R_{2 n+1}^{A}=\mathfrak{l}_{2 n+1}^{A}$.

Definition 4.12. We define $\left(R_{2 n+1}^{A}, \triangleright\right)$ as the Heisenberg rack.

We want to explicit that the multiplication $\triangleright$ in $R_{2 n+1}^{A}$ is a perturbation of the conjugation of the Heisenberg Lie group $H_{2 n+1}$. To do this, we will use the canonical matrix representation

$$
H_{2 n+1}=\left\{\left(\begin{array}{ccc}
1 & x & z \\
0 & I_{n} & y^{t} \\
0 & 0 & 1
\end{array}\right) \mid x=\left(x_{1}, \ldots, x_{n}\right), y=\left(y_{1}, \ldots, y_{n}\right) \in \mathbb{R}^{n}, z \in \mathbb{R}\right\} \leq \mathrm{GL}_{n+2}(\mathbb{R}) .
$$

The conjugation formula for two matrices in $H_{2 n+1}$ is given by

$$
\left(\begin{array}{ccc}
1 & x & z \\
0 & I_{n} & y^{t} \\
0 & 0 & 1
\end{array}\right)\left(\begin{array}{ccc}
1 & x^{\prime} & z^{\prime} \\
0 & I_{n} & y^{\prime t} \\
0 & 0 & 1
\end{array}\right)\left(\begin{array}{ccc}
1 & x & z \\
0 & I_{n} & y^{t} \\
0 & 0 & 1
\end{array}\right)^{-1}=\left(\begin{array}{ccc}
1 & x^{\prime} & z^{\prime}+\sum_{i=1}^{n}\left(x_{i} y_{i}^{\prime}-y_{i} x_{i}^{\prime}\right) \\
0 & I_{n} & y^{\prime t} \\
0 & 0 & 1
\end{array}\right)
$$

With the same representation, the multiplication $\triangleright$ of $R_{2 n+1}^{A}$ turns into

$$
\left(\begin{array}{ccc}
1 & x & z \\
0 & I_{n} & y^{t} \\
0 & 0 & 1
\end{array}\right) \triangleright\left(\begin{array}{ccc}
1 & x^{\prime} & z^{\prime} \\
0 & I_{n} & y^{\prime t} \\
0 & 0 & 1
\end{array}\right)=\left(\begin{array}{ccc}
1 & x^{\prime} & z^{\prime}+\sum_{i, j=1}^{n}\left[\left(\delta_{i j}+a_{i j}\right) x_{i} y_{j}^{\prime}+\left(\delta_{i j}-a_{i j}\right) x_{i}^{\prime} y_{j}\right] \\
0 & I_{n} & y^{\prime t} \\
0 & 0 & 1
\end{array}\right)
$$

hence for $A=0_{n \times n}$, it holds $R_{2 n+1}^{0_{n \times n}}=\operatorname{Conj}\left(H_{2 n+1}\right)$.

Example 4.13. Let $\mathfrak{g}=\mathfrak{k}_{n}$ and let $\left\{e_{1}, \ldots, e_{n}, f_{1}, \ldots, f_{n}, h\right\}$ be a basis of $\mathfrak{g}$. Then the Leibniz bracket of $\mathfrak{g}$ is given by

$\left[\left(x_{1}, \ldots, x_{n}, y_{1}, \ldots, y_{n}, z\right),\left(x_{1}^{\prime}, \ldots, x_{n}^{\prime}, y_{1}^{\prime}, \ldots, y_{n}^{\prime}, z^{\prime}\right)\right]=\left(0, \ldots, 0, x_{1} y_{1}^{\prime}+\sum_{i=2}^{n}\left(x_{i} y_{i}^{\prime}+x_{i} y_{i-1}^{\prime}+x_{i-1}^{\prime} y_{i-1}-x_{i}^{\prime} y_{i-1}\right)+x_{n}^{\prime} y_{n}\right)$

so we obtain a Lie global rack $K_{n}=\left(\mathfrak{g}_{0} \times_{f} \mathbb{R} h, \triangleright\right)$ with multiplication

$$
\begin{aligned}
\left(x_{1}, \ldots, x_{n}, y_{1}, \ldots, y_{n}, z\right) \triangleright\left(x_{1}^{\prime}, \ldots, x_{n}^{\prime}, y_{1}^{\prime}, \ldots, y_{n}^{\prime}, z^{\prime}\right) & = \\
& =\left(x_{1}^{\prime}, \ldots, x_{n}^{\prime}, y_{1}^{\prime}, \ldots, y_{n}^{\prime}, z^{\prime}+x_{1} y_{1}^{\prime}+\sum_{i=2}^{n}\left(x_{i} y_{i}^{\prime}+x_{i} y_{i-1}^{\prime}+x_{i-1}^{\prime} y_{i-1}-x_{i}^{\prime} y_{i-1}\right)+x_{n}^{\prime} y_{n}\right)
\end{aligned}
$$

and $T_{(0,0)} K_{n}=\mathfrak{k}_{n}$. 
Definition 4.14. We define $\left(K_{n}, \triangleright\right)$ as the Kronecker rack.

Example 4.15. Let $\mathfrak{g}=\mathfrak{d}_{n}$ and let $\left\{e_{1}, \ldots, e_{2 n+1}, h\right\}$ be a basis of $\mathfrak{g}$. Then the Leibniz bracket of $\mathfrak{g}$ is given by

$$
\left[\left(x_{1}, \ldots, x_{2 n+1}, z\right),\left(x_{1}^{\prime}, \ldots, x_{2 n+1}^{\prime}, z^{\prime}\right)\right]=(0, \ldots, 0, \bar{z}),
$$

where

$$
\bar{z}=x_{1} x_{n+2}^{\prime}+\sum_{i=2}^{n} x_{i}\left(x_{i+n}^{\prime}+x_{i+n+1}^{\prime}\right)+x_{n+1} x_{2 n+1}^{\prime}+\sum_{i=n+2}^{2 n+1} x_{i}\left(x_{i-n}^{\prime}-x_{i-n-1}^{\prime}\right),
$$

thus a Lie global rack integrating $\mathfrak{g}$ is $D_{n}=\left(\mathfrak{g}_{0} \times_{f} \mathbb{R} h, \triangleright\right)$ with multiplication

$$
\left(x_{1}, \ldots, x_{n}, y_{1}, \ldots, y_{n}, z\right) \triangleright\left(x_{1}^{\prime}, \ldots, x_{n}^{\prime}, y_{1}^{\prime}, \ldots, y_{n}^{\prime}, z^{\prime}\right)=\left(x_{1}^{\prime}, \ldots, y_{1}^{\prime}, \ldots, y_{n}^{\prime}, z^{\prime}+\bar{z}\right)
$$

and $T_{(0,0)} D_{n}=\mathfrak{d}_{n}$.

Definition 4.16. We call $\left(D_{n}, \triangleright\right)$ the Dieudonné rack.

We want co conclude our paper with the following example. The realification of an indecomposable nilpotent Leibniz algebra with one-dimensional commutator ideal over the field $\mathbb{C}$ is a nilpotent real Leibniz algebra with two-dimensional commutator ideal. In the following example, we integrate the realification of the complex indecomposable Heisenberg Leibniz algebra $\mathfrak{l}_{2 n+1}^{J_{a}}$, where $J_{a} \in \mathrm{M}_{n}(\mathbb{C})$ is the Jordan block of eigenvalue $a \in \mathbb{C}$.

Example 4.17. Let $\mathfrak{h}=\mathfrak{l}_{2 n+1}^{J_{a}}$ and let $\left\{e_{1}, \ldots, e_{n}, f_{1}, \ldots, f_{n}, h\right\}$ be a basis of $\mathfrak{h}$ over $\mathbb{C}$. Then $\operatorname{dim}_{\mathbb{R}} \mathfrak{h}=4 n+2$ and $\left\{e_{1}, i e_{1}, \ldots, e_{n}, i e_{n}, f_{1}, i f_{1}, \ldots, f_{n}, i f_{n}, h, i h\right\}$ is a basis of $\mathfrak{h}$ over $\mathbb{R}$. For every $\left(x_{1}, \ldots, x_{n}, y_{1}, \ldots, y_{n}, z\right)$, $\left(x_{1}^{\prime}, \ldots, x_{n}^{\prime}, y_{1}^{\prime}, \ldots, y_{n}^{\prime}, z^{\prime}\right) \in \mathbb{C}^{2 n+1}$, the Leibniz bracket of $\mathfrak{h}$ over $\mathbb{R}$ is given by

$$
\left[\left(x_{1}, \ldots, x_{n}, y_{1}, \ldots, y_{n}, z\right),\left(x_{1}^{\prime}, \ldots, x_{n}^{\prime}, y_{1}^{\prime}, \ldots, y_{n}^{\prime}, z^{\prime}\right)\right]=(0, \ldots, 0, \Re(\bar{z}), \Im(\bar{z})),
$$

where $\Re(a+i b)=a, \Im(a+i b)=b$ and

$$
\bar{z}=\sum_{i=1}^{n}\left[(1+a) x_{i} y_{i}^{\prime}+(-1+a) x_{i}^{\prime} y_{i}\right]+\sum_{i=2}^{n}\left(x_{i} y_{i-1}^{\prime}+x_{i}^{\prime} y_{i-1}\right)
$$

Thus a Lie global rack integrating $(\mathfrak{h},[-,-])$ is $\left(\mathfrak{h}_{0} \times_{f} \operatorname{Span}_{\mathbb{R}}\{h, i h\}, \triangleright\right)$ with multiplication

$$
\begin{aligned}
& \left(x_{1}, \ldots, x_{n}, y_{1}, \ldots, y_{n}, z\right) \triangleright\left(x_{1}^{\prime}, \ldots, x_{n}^{\prime}, y_{1}^{\prime}, \ldots, y_{n}^{\prime}, z^{\prime}\right)= \\
& =\left(\Re\left(x_{1}^{\prime}\right), \Im\left(x_{1}^{\prime}\right), \ldots, \Re\left(x_{n}^{\prime}\right), \Im\left(x_{n}^{\prime}\right), \Re\left(y_{1}^{\prime}\right), \Im\left(y_{1}^{\prime}\right), \ldots, \Re\left(y_{n}^{\prime}\right), \Im\left(y_{n}^{\prime}\right), \Re\left(z^{\prime}+\bar{z}\right), \Im\left(z^{\prime}+\bar{z}\right)\right) .
\end{aligned}
$$

\section{References}

[1] J.-L. Loday. "Une version non commutative des algebres de Lie: les algebres de Leibniz". In: Les rencontres physiciens-mathématiciens de Strasbourg-RCP25 44 (1993), pp. 127-151.

[2] A. Blokh. "A generalization of the concept of a Lie algebra". In: Dokl. Akad. Nauk SSSR 165.3 (1965), pp. $471-473$.

[3] G. Falcone and Á. Figula. "The action of a compact Lie group on nilpotent Lie algebras of type $\{\{n, 2\}\}$ ". In: Forum Mathematicum. Vol. 28. 4. De Gruyter. 2016, pp. 795-806.

[4] C. Bartolone, A. Di Bartolo, and G. Falcone. "Nilpotent Lie algebras with 2-dimensional commutator ideals". In: Linear algebra and its applications 434.3 (2011), pp. 650-656.

[5] S. Ayupov, B. Omirov, and I. Rakhimov. Leibniz Algebras: Structure and Classification. ISBN: 9781000740004.

[6] S. Mac Lane. Categories for the working mathematician. Vol. 5. Springer Science \& Business Media, 2013.

[7] L. Kronecker. Algebraische Reduktion der Scharen bilinearer Formen, Sitzungsberichte Akad. 1890. 
[8] J. Dieudonné. "Sur la réduction canonique des couples de matrices". fr. In: Bulletin de la Société Mathématique de France 74 (1946), pp. 130-146. DOI: 10.24033/bsmf.1380.

[9] G. Falcone and M. A. Vaccaro. "Kronecker modules and reductions of a pair of bilinear forms". eng. In: Acta Universitatis Palackianae Olomucensis. Facultas Rerum Naturalium. Mathematica 1 (), pp. 55-60.

[10] N. Jacobson. Basic Algebra I: Second Edition. Dover Books on Mathematics. Dover Publications, 2012. ISBN: 9780486135229. URL: https://books.google.it/books?id=JHFpv0tKiBAC.

[11] M.-K. Kinyon. "Leibniz algebras, Lie racks, and digroups". In: J. Lie Theory, 17(1):99-114 (2007).

[12] S. Covez. "The local integration of Leibniz algebras". In: Annales de l'Institut Fourier. Vol. 63. 1. 2013, pp. $1-35$.

[13] M. Bordemann and F. Wagemann. "A dirty integration of Leibniz algebras". In: Journal of Lie Theory 27 (June 2016).

[14] J. Mostovoy. "A Comment on the Integration of Leibniz Algebras". In: Communications in Algebra 41.1 (2013), pp. 185-194. DOI: 10.1080/00927872.2011.625562. 